\title{
Module Production and Qualification for the Phase I Upgrade of the CMS Pixel Detector
}

\section{Benedikt Freund ${ }^{* \dagger}$}

KIT - Karlsruhe Institute of Technology (DE)

E-mail: benedikt. freundecern.ch

\begin{abstract}
After consolidation of the LHC in 2013/14 its centre-of-mass energy will increase to $13 \mathrm{TeV}$ and the luminosity will reach $2 \cdot 10^{34} \mathrm{~cm}^{-2} \mathrm{~s}^{-1}$, which is twice the design luminosity. The latter will result in more simultaneous particle collisions, which would significantly increase the dead time of the current readout chip of the CMS pixel detector. Therefore the entire CMS pixel detector is replaced in 2016/17 and a new digital readout with larger buffers will be used to handle increasing pixel hit rates. An additional fourth barrel-layer provides more space points to improve track reconstruction. Half of the required modules for layer four is being produced at Karlsruhe Institute of Technology (KIT). This poster/talk deals with the smallest discrete subunit of the pixel detector, the module and its assembly process. Moreover first production experience will be shown.
\end{abstract}

The European Physical Society Conference on High Energy Physics

22-29 July 2015

Vienna, Austria

*Speaker.

$\dagger$ on behalf of the CMS Collaboration 


\section{Introduction}

The Phase I Upgrade of the CMS pixel detector comprises several improvements affecting its technology and geometry. Regarding the barrel region there are two major modifications, on the one hand there will be an additional fourth layer providing one more space point for the tracking. On the other hand the reconstruction of vertices is improved - which is for instance important for b-tagging - by moving the innermost layer closer to the interaction point. This became possible as a smaller beam pipe was installed (in the detector regions) during the consolidation of the LHC in 2013/14. A comparison illustrating the differences of the barrel region of the current and future CMS pixel detector is presented in Figure 1.

A further important change is the redesign of the cooling system. Due to that the material budget in the sensitive area will be reduced, despite adding a layer to the barrel region. In detail this is realised by replacing the monophase $\mathrm{C}_{6} \mathrm{~F}_{14}$ cooling by a two-phase $\mathrm{CO}_{2}$ cooling that allows to use less coolant and smaller cooling pipes [1].

Additional enhancements are related to the new ROC, addressing - in particular - the integrated buffers, since the current version of the ROC has too few to buffer all events at a pile-up of 50. Hence the number of data and time stamp buffers is increased from 32 to 80 respectively from 12 to 24 . Due to the increased number of modules it is also necessary to raise bandwidth which is achieved by using digital decoded readout instead of the current analogue readout to assure that the increased amount of data can be sent out of the detector in time [2].

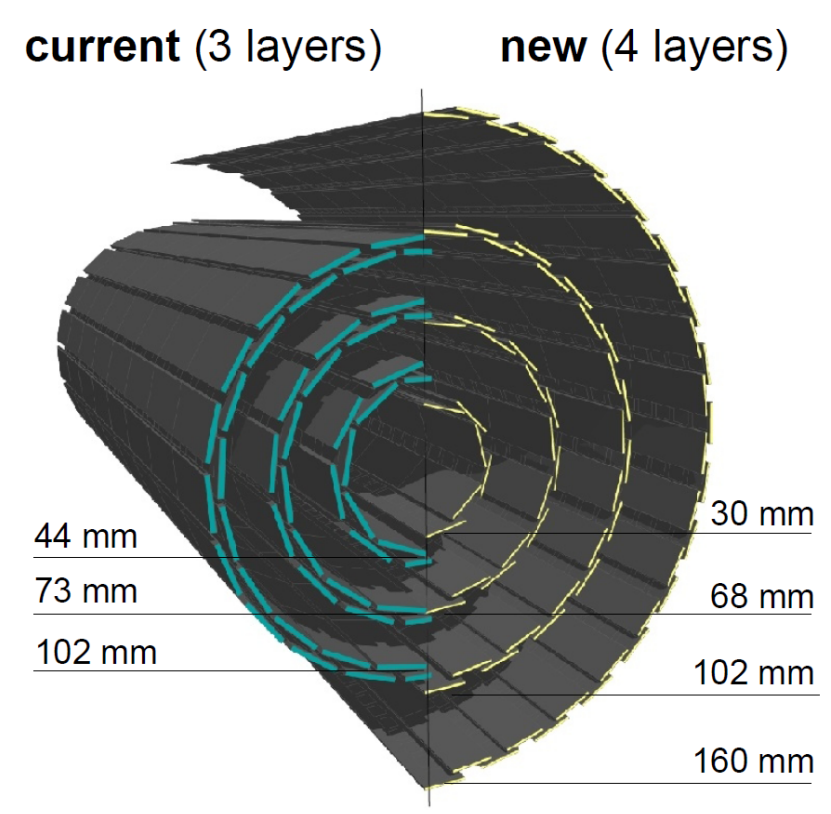

Figure 1: Comparison of the barrel region of the CMS pixel detector before and after the Phase I Upgrade. The current pixel detector consists of three layers, while the Phase I pixel detector will be equipped with an additional layer. In addition layer one and two of the new pixel detector will be mounted closer to the interaction point [1]. 


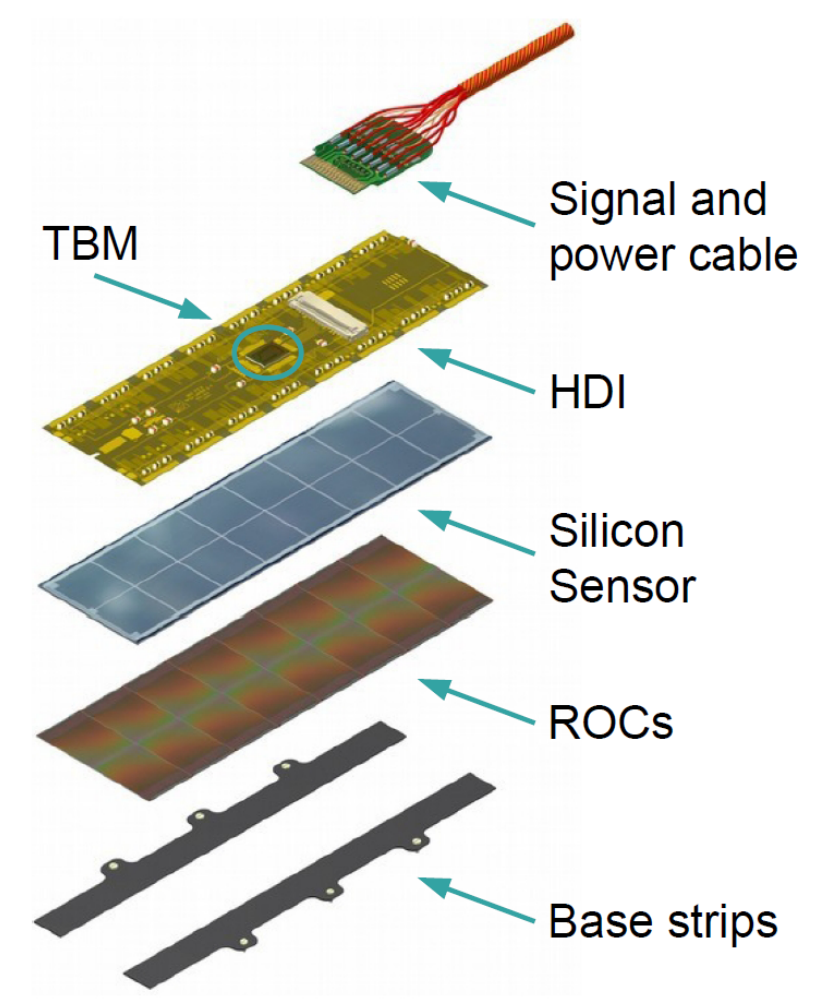

Figure 2: Exploded view of a CMS pixel detector module. A module consists of a silicon sensor, where signals are generated, and 16 ROCs collecting the charge and processing the signals. Additional components like HDI, TBM and a cable are used to connect and control the ROCs and to send all data out of the detector. Two base strips are used to fix the modules on the carbon fibre support structure [1].

\section{CMS Pixel Detector Modules}

A CMS pixel detector module is the smallest subunit and consists of several components that are shown in the exploded view in Figure 2. In the centre of the module is the $n^{+}$-in- $n$ silicon sensor, where electron-hole pairs are generated once a charged particle traverses. Below the sensor - referring to the Figure - are 16 ROCs with 66560 pixels $\left(150 \times 100 \mu \mathrm{m}^{2}\right.$ each $)$ in total. Each pixel is connected via a bump-bond to the corresponding pixel on the sensor side and via these bump-bonds the signals generated in the sensor are read out and subsequently processed and stored in the ROCs. Two base strips are glued to the backside of the ROCs and serve to fix the module on support structures once they are mounted in the experiment. Onto the top of the silicon sensor a high density interconnect $(H D I)$ is glued providing connections between the 16 ROCs and the token bit manager chip (TBM). The TBM, which is located in the centre of the HDI, controls all ROCs of a module, collects their data and sends it out of the detector. The 16 ROCs as well as the TBM are connected to the HDI via wire bonds. In addition the HDI is equipped with a connector matching a twisted pair signal and power cable [3]. 


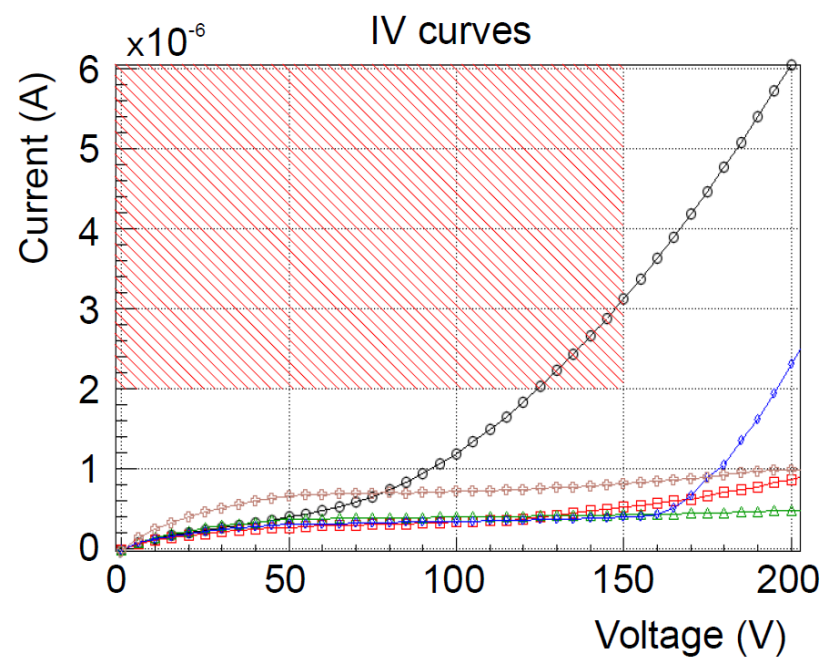

Figure 3: Current-voltage characteristics of five silicon sensors. The black line crosses the red dashed area, which means that this sensor has more than $2 \mu \mathrm{A}$ at $150 \mathrm{~V}$ and hence fails the electrical test. Further the black line has a slope larger than 2 between $100 \mathrm{~V}$ and $150 \mathrm{~V}$ which is also out of the required specifications. The four remaining IV characteristics pass both criteria.

Since all components undergo various external handling and processing steps before they arrive at the production centres a detailed inspection is indispensable. The same is true for all intermediate products to identify problems within the production chain. Among these quality checks are optical inspections to detect all kind of physical damage as well as electrical and X-ray tests to examine the functionality.

\subsection{Silicon Sensors}

The properties of the silicon sensors have huge influence on the performance of the pixel detector, hence they are checked optically as well as electrically. During the optical tests the sensors are screened for physical damage like scratches as these also can have long-term effects on initially good sensors. The crucial feature of sensors are their electrical properties which are determined by the current-voltage $(I V)$ characteristics, where two grading criteria have to be fulfilled:

- $\mathrm{I}(150 \mathrm{~V})<2 \mu \mathrm{A}$,

- $\frac{\mathrm{I}(150 \mathrm{~V})}{\mathrm{I}(100 \mathrm{~V})}<2$.

Exemplary results of five sensors are presented in Figure 3, where the black line represents a sensor which fails both criteria. Its IV characteristics crosses the red dashed area and thus has more than $2 \mu \mathrm{A}$ at $150 \mathrm{~V}$. Aside from that the black line has a quotient larger than 2 failing also the second criteria. The remaining four sensors fulfil both criteria and will be used in the production.

\subsection{Readout Chips}

Testing the readout chips demands a reliable identification of missing or defective bumps since a good bump-bond connection to the sensor is essential to collect the charge generated by traversing 


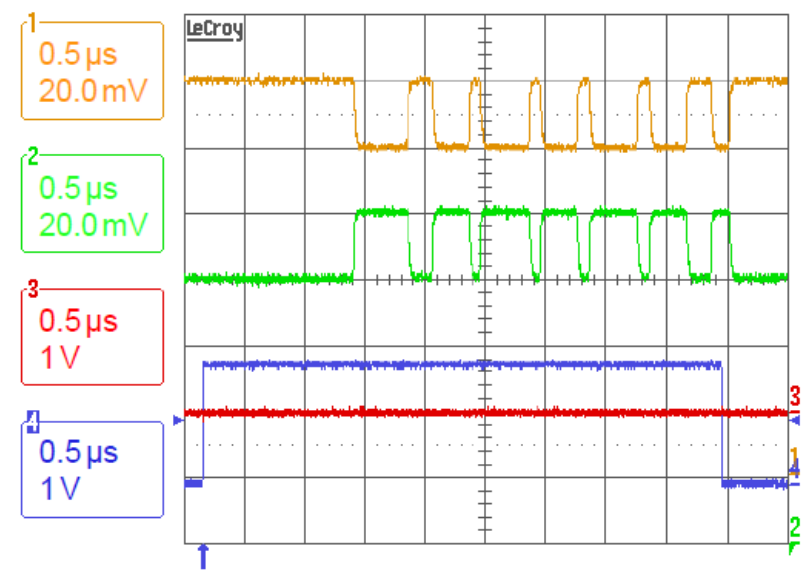

Figure 4: Measured TBM test signals. The yellow and green lines are the signals of two measured data lines and are the ones of interest. The patterns of these two signals have to be compared by eye to a control pattern while the signal height is directly visible on the oscilloscope interface. Both signals have a signal height of $20 \mathrm{mV}$ and clearly fulfil the minimal requirement of $15.5 \mathrm{mV}$.

particles reliably. A single ROC has 4160 pixels and each pixel is equipped with a bump which is inspected via an automated pattern recognition. That pattern recognition is able to detect too small, too big, non circular and missing bumps as well as scratches or other suspicious areas on the ROC. Exclusively readout chips without scratches and less than $1 \%$ problematic bumps are processed further.

\subsection{High Density Interconnect and Token Bit Manager}

HDI and TBM are individually optically inspected for physical flaws (scratches, cracks and missing passive components or discolourations on the HDI) and electrically tested once they are glued together and wire bonded. Within the electrical test certain test signals are send to the TBM, via a cable connected to the HDI, and read out via a needle card connected to the HDI. The outgoing signals are checked with an oscilloscope where the signal pattern and heights have to match certain specifications. While the signal patterns have to be compared by eye to a control pattern the minimum signal height of $15.5 \mathrm{mV}$ is directly checked via the oscilloscope as shown in Figure 4.

\subsection{Bare Module}

A bare module is an intermediate product, consisting of a sensor connected to 16 ROCs, which requires several tests to determine its quality. First the sensor is screened again for scratches which may have occurred during the flip-chip process. The electrical test contains a repetition of the measurement of the IV characteristic of the sensor to verify that the electrical properties did not change due to the flip-chipping, as well as a programmability check of the ROCs plus a functionality test where each pixel cell and bump-bond connection is tested. In detail the functionality check of the ROCs is realised by sending a command to tune a certain current of the ROC and measuring the difference. Afterwards all 4160 pixels of each ROC are tested by injecting a calibration pulse into each of them and by comparing the number of registered pulses to the number of injected pulses. The final electrical test of a bare module is to check each bump-bond connection by injecting - pixel 


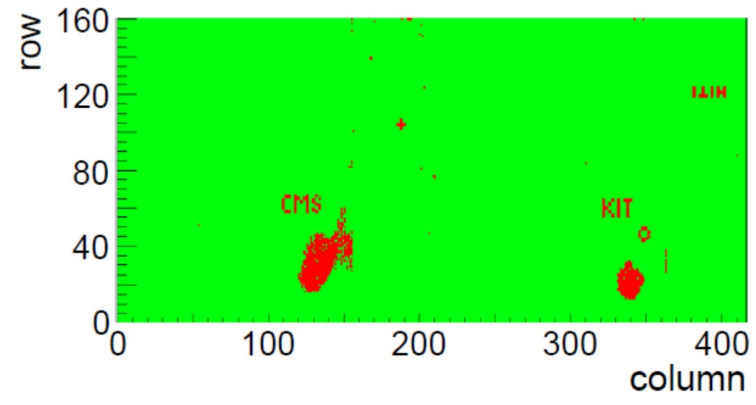

(a) Bump-Bond Test

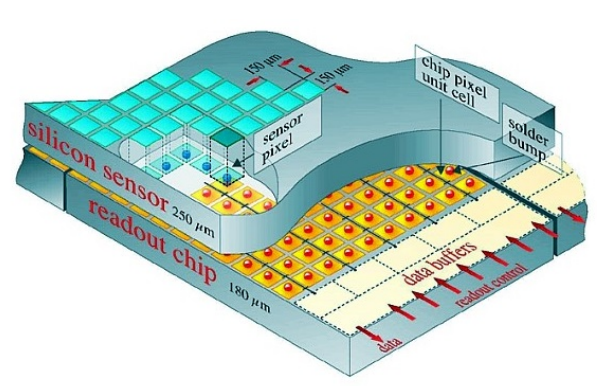

(b) Bare Module

Figure 5: Figure (a) shows the result of a bump-bond test. Red areas represent defective bump-bond connections, which were removed on purpose beforehand to examine the reliability of the bump-bond test. Figure (b) illustrates the structure of a bare module. All pixels of sensor and ROCs are connected via a bump-bond depicted as red dots [4] [5].

by pixel - calibration pulses capacitively from ROC into sensor. If the bump-bond connection is not intact the generated charge cannot be read out and a defect bump-bond connection is identified. Figure 5 shows the result of a bump-bond test of a bare module and a schematic cross section. The red dots in the bump-bonding test show defect bump-bond connections which - recognisable by the shape of the red areas - were removed on purpose to verify the reliability of the bump-bond test. The schematic indicates the structure of a bare module with each pixel of ROC and sensor being connected via a bump-bond.

\subsection{Full Module}

The final step in the assembly process is to glue the HDI - already equipped with a TBM and the base strips to the bare module and to place the wire bond connections from the HDI to the ROCs. Afterwards a final optical inspection is performed, followed by electrical tests including an IV characteristics and functionality checks as executed in the bare module test. The final test exploits X-rays to generate charge by photons hitting the sensor, and reading out the signal via the entire readout chain of a full module. In this way, reliable information is obtained on which pixels are working as desired and which are defect or have a broken bump-bond connection. In Figure 6 a measurement with X-rays and a picture of a full module are shown. It is evident that larger components - like the cable, the connector or capacitors - on the module (see picture 6 (b)) correspond to areas with less hits in the measurement since larger components shield the sensor from some photons.

After a module passes the final test it is shipped to the next center where more detailed measurements are performed. These measurements include thermal cycling, a highrate test and a calibration curve using monochromatic X-rays. The very last step is the mounting of the modules onto the carbon fibre support structures before they are installed into the CMS detector. 


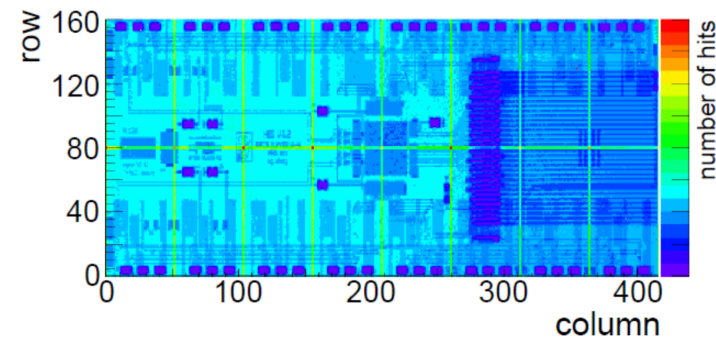

(a) X-ray Measurement

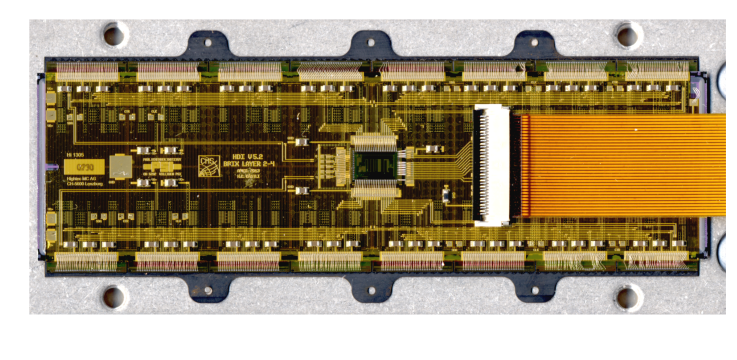

(b) Full Module

Figure 6: Figure (a) shows a measurement with X-rays. White dots reveal defect pixel cells or bump connections, while areas with less entries clearly indicate the position of the connector, the cable, the TBM and several capacities preventing some photons from reaching the sensor. Figure (b) shows a picture of a fully assembled module. Comparing the picture to the measurement shows that the location of the larger components match.

\section{Outlook}

The module production and qualification for layer two, three and four started in 2015 and will end in early 2016. In case of the innermost layer the production is scheduled slightly later when modified ROCs, TBMs and HDIs are delivered which can handle the highest rates closest to the interaction point. Since for layer one the fewest modules are required this fits into the production timetable for the remaining layers. Once the production is completed and the modules are qualified they will be mounted on support structures to be ready for installation into CMS during the extended year end technical stop 2016/17.

\section{References}

[1] CMS Collaboration, CMS Technical Design Report for the Pixel Detector Upgrade, CERN-LHCC-2012-016 (2012).

[2] F. Meier, psi46dig pixel chip External Specification Manual (2013).

[3] L. Caminada und A. Starodumov, Building and commissioning of the CMS pixel barrel detector, JINST 4 (2009) P03017Journal of Instrumentation, 4(03):P03017 (2009).

[4] B. Hiti, Probe Station for Functionality Tests of Bare Modules for the Phase I Upgrade of the CMS Pixel Detector, Karlsruhe Institute of Technology, master thesis, IEKP-KA/2015-13 (2015).

[5] CMS Collaboration, http://cms.web.cern.ch/news/silicon-pixels (Oct. 04, 2015). 